\title{
Origin of change in molecular-weight dependence for polymer surface tension
}

\author{
R. B. Thompson, ${ }^{1}$ J. R. MacDonald, ${ }^{2}$ and P. Chen ${ }^{3}$ \\ ${ }^{1}$ Department of Physics and Astronomy, University of Waterloo, Waterloo, Ontario, Canada N2L $3 G 1$ \\ ${ }^{2}$ Department of Electrical and Computer Engineering, University of Waterloo, Waterloo, Ontario, Canada N2L $3 G 1$ \\ ${ }^{3}$ Department of Chemical Engineering, University of Waterloo, Waterloo, Ontario, Canada N2L 3G1 \\ (Received 11 July 2007; revised manuscript received 13 August 2008; published 30 September 2008)
}

\begin{abstract}
Self-consistent-field theory is used to reproduce the behavior of polymer surface tension with molecularweight for both lower and higher molecular-weight polymers. The change in behavior of the surface tension between these two regimes is shown to be due to the almost total exclusion of polymer from the nonpolymer bulk phase. The predicted two regime surface tension behavior with molecular-weight and the exclusion explanation are shown to be valid for a range of different polymer compressibilities.
\end{abstract}

DOI: 10.1103/PhysRevE.78.030801 PACS number(s): 36.20. - r, 68.03.Cd, 71.15.Mb, 82.70.Rr

An understanding of polymer surface tension is vital for many industrial processes such as foaming, suspensions, wetting, and blending [1]. Predictions for foaming processes depend on classical nucleation theory which in turn requires values of surface tension as input. As a result, efforts to improve the measurement and understanding of polymer surface tensions have been ongoing for many years. In theoretical studies, the molecular-weight (MW) dependence of polymer surface tension is particularly important since higher MW polymers offer challenges for accurate experimental measurements. Although progress has been made in experimentally measuring surface tensions of high MW polymers (see Ref. [2]), theoretical verifications and explanations for observed behaviors are lacking. That being said, some important contributions related to this subject have been made; see, for example, Poser and Sanchez [3], Helfand, Bhattacharjee, and Fredrickson [4], Ermoshkin and Semenov [5], and Kumar and Jones [6].

In this paper, we show that many approximations made in previous works [3-6] are unnecessary as the polymer surface tension can be calculated using full numerical selfconsistent-field theory (SCFT) with no approximations beyond the mean field. SCFT is an appropriate method to use to study polymer surface tension because although SCFT is a coarse-grained theory, it is microscopic in the sense that it includes all polymer configurational degrees of freedom, unlike phenomenological density gradient theory, for example, [7-9]. Jones and Richards [10] point out that presently polymer surface tension MW dependence is "explained" in terms of a mix of phenomenological density gradient theory for higher MW and an empirical formula for lower MW. This is a wholly unsatisfactory situation. We will show that SCFT spontaneously reproduces the expected experimental two regime behavior of polymer surface tension, and allows us to explain why there is a change of behavior between low and high MW polymer surface tensions. Since an understanding of MW dependence of surface tension is an important ingredient for the advancement of many industrial processes, these SCFT results represent an important step beyond previous theories.

The SCFT formalism for polymer surface tension has been described by us in a previous presentation [11] where it was successfully used to predict qualitative surface tension trends as a function of temperature and pressure for a single polymer MW. There we used a parameter $\alpha=0.1 ; \alpha$ is the ratio of the volume of a solvent molecule to the volume of a polymer molecule. It is inversely proportional to the polymer MW. In the present work, we lower the value of $\alpha$ (increase the polymer MW) to investigate the behavior of surface tension as a function of MW. The free energy for a compressible polymer-solvent system is

$$
\begin{aligned}
\frac{N F}{\rho_{0} k_{B} T V}= & -\frac{\phi_{s}}{\alpha} \ln \left(\frac{Q_{s} \alpha}{V \phi_{s}}\right)-\frac{\phi_{h}}{\alpha} \ln \left(\frac{Q_{h} \alpha}{V \phi_{h}}\right)-\phi_{p} \ln \left(\frac{Q_{p}}{V \phi_{p}}\right) \\
& +\frac{1}{V} \int d \mathbf{r}\left(\chi_{p s} N \varphi_{p}(\mathbf{r}) \varphi_{s}(\mathbf{r})+\frac{1}{2} \chi_{s s} N \varphi_{s}(\mathbf{r}) \varphi_{s}(\mathbf{r})\right. \\
& +\frac{1}{2} \chi_{p p} N \varphi_{p}(\mathbf{r}) \varphi_{p}(\mathbf{r})-w_{s}(\mathbf{r}) \varphi_{s}(\mathbf{r})-w_{h}(\mathbf{r}) \varphi_{h}(\mathbf{r}) \\
& \left.-w_{p}(\mathbf{r}) \varphi_{p}(\mathbf{r})-\xi(\mathbf{r})\left[1-\varphi_{s}(\mathbf{r})-\varphi_{h}(\mathbf{r})-\varphi_{p}(\mathbf{r})\right]\right),
\end{aligned}
$$

where $\varphi_{s}(\mathbf{r}), \varphi_{p}(\mathbf{r})$, and $\varphi_{h}(\mathbf{r})$ are the local volume fractions of solvent, polymer, and "holes," respectively, and $\omega_{s}(\mathbf{r})$, $\omega_{p}(\mathbf{r})$, and $\omega_{h}(\mathbf{r})$ are conjugate chemical potential fields. The function $\xi(\mathbf{r})$ is a pressure field that enforces a constant total density, including the holes. Compressible systems with inhomogeneous total densities are thus modeled in this incompressible formalism through the presence or absence of vacancies (holes). This is a method introduced by Hong and Noolandi that reduces to the Sanchez-Lacombe equation of state in the limit of a homogeneous system [12]. Other equations of state can also be chosen within the SCFT formalism [13]. $Q_{s}$ and $Q_{p}$ are the partition functions for single molecules of solvent and polymer, respectively, subject to the fields $\omega_{s}(\mathbf{r})$ and $\omega_{p}(\mathbf{r})$, while an analogous role is played by $Q_{h}$ for the holes. $\phi_{s}, \phi_{p}$, and $\phi_{h}$ are the global volume fractions of solvent, polymer, and holes, respectively, in the system. The $\chi$ parameters are the Flory-Huggins representations of the interactions between solvent and polymer segments based on a segment volume $\rho_{0}^{-1}$, taken to be the same for all constituents. With respect to this segment volume, a polymer has a degree of polymerization $N$ and $\alpha$ becomes simply $1 / N$. Note that $\alpha$ can be more complicated if different segment volumes are used for the various constituents and so we will continue to refer to $\alpha$ in some places in the interest of generality of formalism. We have presented a free energy 
density (1) by dividing $F$ by the system volume $V$. This free energy density is made dimensionless by dividing by the thermal energy $k_{B} T$, where $k_{B}$ is Boltzmann's constant and $T$ is the temperature, and multiplying by the volume of a single polymer, $N / \rho_{0}$. Since we are to compare the surface tensions of a number of different MW polymers, we choose a reference $N_{0}$ corresponding to the value $N_{0}=10 \quad(\alpha=0.1)$. The surface tension (interfacial tension between polymer and solvent) $\gamma$ is found from the excess free energy $F_{\text {ex }}$ through $\gamma$ $=F_{\text {ex }} / A$ where $A$ is the surface area between the phases. The excess free energy is the free energy of the entire system minus the free energies of the bulk system on either side of the interface $F_{\text {ex }}=F-F_{s}^{\text {bulk }}-F_{p}^{\text {bulk }}-F_{h}^{\text {bulk }}[14,15]$. Here, $F_{i}^{\text {bulk }}$ for $i=s, p, h$ can be found from Eqs. (14)-(18) of Ref. [11]. The area $A$ can be replaced with $V / L$ where $L$ is the extent of the simulation in the direction perpendicular to $A$; it must be chosen large enough so that bulk conditions are reached on both sides of the interface. To make this surface tension dimensionless, the distance $L$ can be divided by a factor $R_{g}^{0}$, the unperturbed radius of gyration of the reference MW polymer with $N=10$. All distances in this work will be measured in terms of this reference radius of gyration. We can also divide the volume $V$ by the volume of the reference polymer, $N_{0} / \rho_{0}$. Last, we can divide the surface tension by the energy $k_{B} T$. The dimensionless surface tension expression then becomes

$$
\frac{R_{g}^{0} \gamma}{a^{2} \rho_{0} k_{B} T}=\left(\frac{L}{R_{g}^{0}}\right) \frac{N_{0} F_{\mathrm{ex}}}{\rho_{0} k_{B} T V},
$$

where $a$ is the statistical segment length of a polymer segment (the average length of a polymer segment). The total system free energy (1) required to calculate the excess free energy (surface tension) is found by varying (1) to give the set of self-consistent equations

$$
\begin{gathered}
w_{s}(\mathbf{r})=\chi_{p s} N \varphi_{p}(\mathbf{r})+\chi_{s s} N \varphi_{s}(\mathbf{r})+\xi(\mathbf{r}), \\
w_{p}(\mathbf{r})=\chi_{p s} N \varphi_{s}(\mathbf{r})+\chi_{p p} N \varphi_{p}(\mathbf{r})+\xi(\mathbf{r}), \\
w_{h}(\mathbf{r})=\xi(\mathbf{r}), \\
\varphi_{s}(\mathbf{r})+\varphi_{p}(\mathbf{r})+\varphi_{h}(\mathbf{r})=1, \\
\varphi_{s}(\mathbf{r})=\frac{\phi_{s} V}{Q_{s}} e^{-\alpha w_{s}(\mathbf{r})}, \\
\varphi_{h}(\mathbf{r})=\frac{\phi_{h} V}{Q_{h}} e^{-\alpha w_{h}(\mathbf{r})}, \\
\varphi_{p}(\mathbf{r})=\frac{\phi_{p} V}{Q_{p}} \int_{0}^{1} d s q(\mathbf{r}, s) q(\mathbf{r}, 1-s),
\end{gathered}
$$

where $q(\mathbf{r}, s)$ is the solution to the modified diffusion equation

$$
\frac{\partial q(\mathbf{r}, s)}{\partial s}=\frac{N a^{2}}{6} \nabla^{2} q(\mathbf{r}, s)-w_{p}(\mathbf{r}) q(\mathbf{r}, s) .
$$

The set of Eqs. (3)-(10) is solved numerically. More information on the theoretical method including details of the

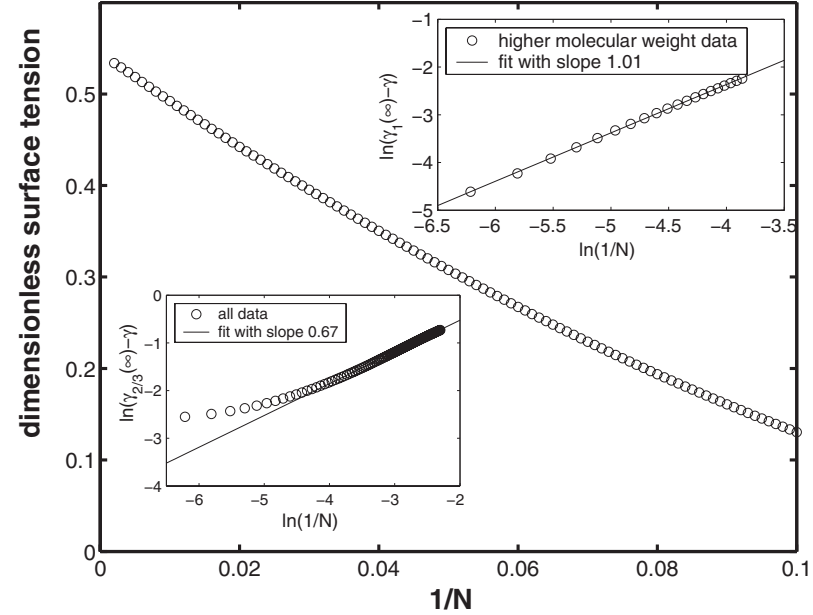

FIG. 1. Dimensionless surface tension as a function of inverse molecular-weight $1 / N$ for an incompressible system (open circles). The lower inset shows the same data plotted logarithmically. The solid line in the lower inset is a fit producing a slope of 0.67 . The upper inset shows a subset of the data corresponding to higher molecular-weights and the line is a fit producing a slope of 1.01 .

excess free energy expression can be found in Ref. [11].

For clarity of discussion, we begin by examining an incompressible system, where $\phi_{h}=0$. Surface tension as a function of $1 / N$ is plotted in Fig. 1 for a system with $\chi N_{0}$ $=10$. The surface tension is seen to increase with increasing MW (decreasing $1 / N$ ) in agreement with experiment $[2,7-9,16,17]$. Further, the surface tension increases according to $\gamma=\gamma(\infty)-c(1 / N)^{x}[c$ is a constant and $\gamma(\infty)$ is the surface tension at infinite molecular-weight] with $x=2 / 3$ for lower MW and $x=1$ for higher MW in agreement with experiment $[7-9,16]$. These trends are shown in the insets of Fig. 1. The lower inset shows the entire data set plotted as $\ln \left[\gamma_{2 / 3}(\infty)-\gamma\right]$ versus $\ln (1 / N)$ where $\gamma_{2 / 3}(\infty)$ is the extrapolated $y$ intercept (infinite MW limit) for the data set based on a linear fitting of the lower MW data only with $(1 / N)^{2 / 3}$. Clearly the lower MW data fit well, producing a slope of 0.666, but higher MW data deviates from the $2 / 3$ behavior, as expected. This higher MW data is therefore plotted in the upper inset as $\ln \left[\gamma_{1}(\infty)-\gamma\right]$ versus $\ln (1 / N)$ where $\gamma_{1}(\infty)$ is the extrapolated $y$ intercept (infinite MW limit) for the data set based on a linear fitting of the higher MW data only with $(1 / N)$. The linear agreement is excellent, producing a slope of 1.015. SCFT therefore shows the same two-regime behavior as has been discussed and debated in the literature [3-10]. SCFT predicts this two-regime behavior spontaneously from a single theory.

To explain why there is a change of behavior in polymer surface tension, the thermodynamic components of the surface tension can be examined. In general, free energies can be written in terms of thermodynamic contributions as $F$ $=U-T S$ where $U$ is the internal energy and $S$ is the entropy of the system. We can therefore break up the surface tension into contributions from the various thermodynamic components $[11,18]$. For an incompressible case we obtain $\gamma=\gamma_{U}$ $+\gamma_{S_{T p}}+\gamma_{S_{C p}}+\gamma_{S_{T S}}$ where the summed terms are the contributions to the surface tension from the internal energy, polymer 


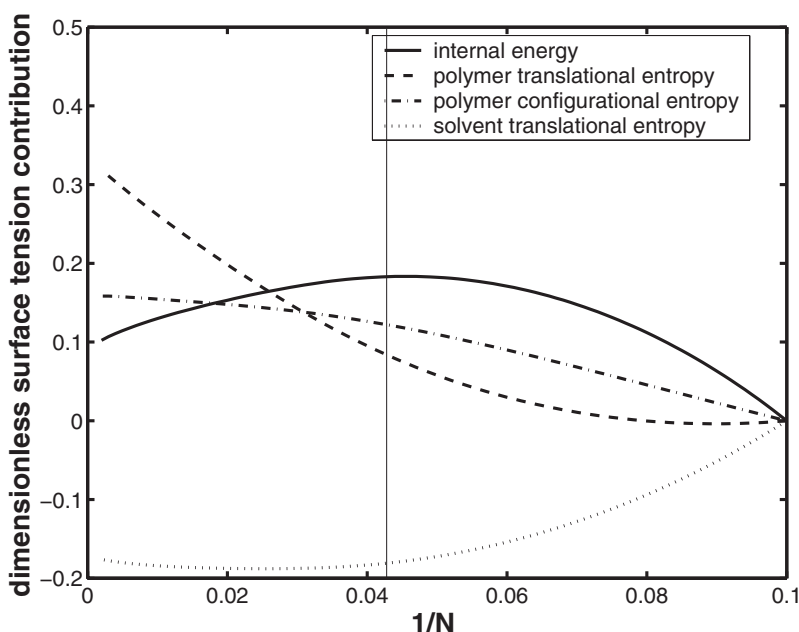

FIG. 2. Thermodynamic components of the dimensionless surface tension as a function of inverse molecular-weight $1 / N$. The legend labels the components. All components have been shifted to zero at $1 / N=0.1$ to better show their behavior (enlarge the scale) as MW is increased. The vertical line shows roughly where the behaviors of the components change.

translational entropy, polymer configurational entropy, and solvent translational entropy, respectively. These components are plotted for the present case in Fig. 2 where they have been shifted along the $y$ axis for clarity. Some components are found to change behavior at around the same place as the total surface tension, as roughly indicated by the vertical line in Fig. 2. In particular, the internal energy contribution changes qualitatively with increasing MW from increasing (right of the vertical line) to decreasing (left of the vertical line). Figure 3 shows the bulk solvent and polymer volume fractions far from the interface. SCFT predicts that the width of the interface between the polymer and solvent becomes more narrow with increasing MW, contributing to a drop in the internal energy of the system. Likewise, from Fig. 3 the amount of polymer in the solvent bulk phase and vice versa decrease with increasing MW so that the internal energy in the bulk regions should also drop. Since $\gamma_{U}$ initially increases in Fig. 2, it must be from the form of $F_{\mathrm{ex}}$ that the bulk internal energy decreases initially at a faster rate than the system as a whole. At some point however, this ceases to be the case, $\gamma_{U}$ starts to decrease, and the surface tension curve changes behavior. From Fig. 3 we see why the bulk internal energy ceases to decrease faster than the system as a whole. The plot of the polymer in the bulk solvent phase [Fig. 3(b)] shows that almost all polymer is eventually excluded from the solvent side of the interface. Beyond this point, the solvent bulk internal energy cannot significantly decrease anymore and the system internal energy starts to decrease more rapidly than the bulk internal energy. In other words, the change of polymer surface tension dependence on MW is a result of reaching the point of almost total exclusion of polymer from the solvent bulk domain.

The incompressible system gives a simple accounting of the physics involved in this polymer surface tension problem but, more realistically, one would prefer to verify that these conclusions are also valid for compressible systems. The
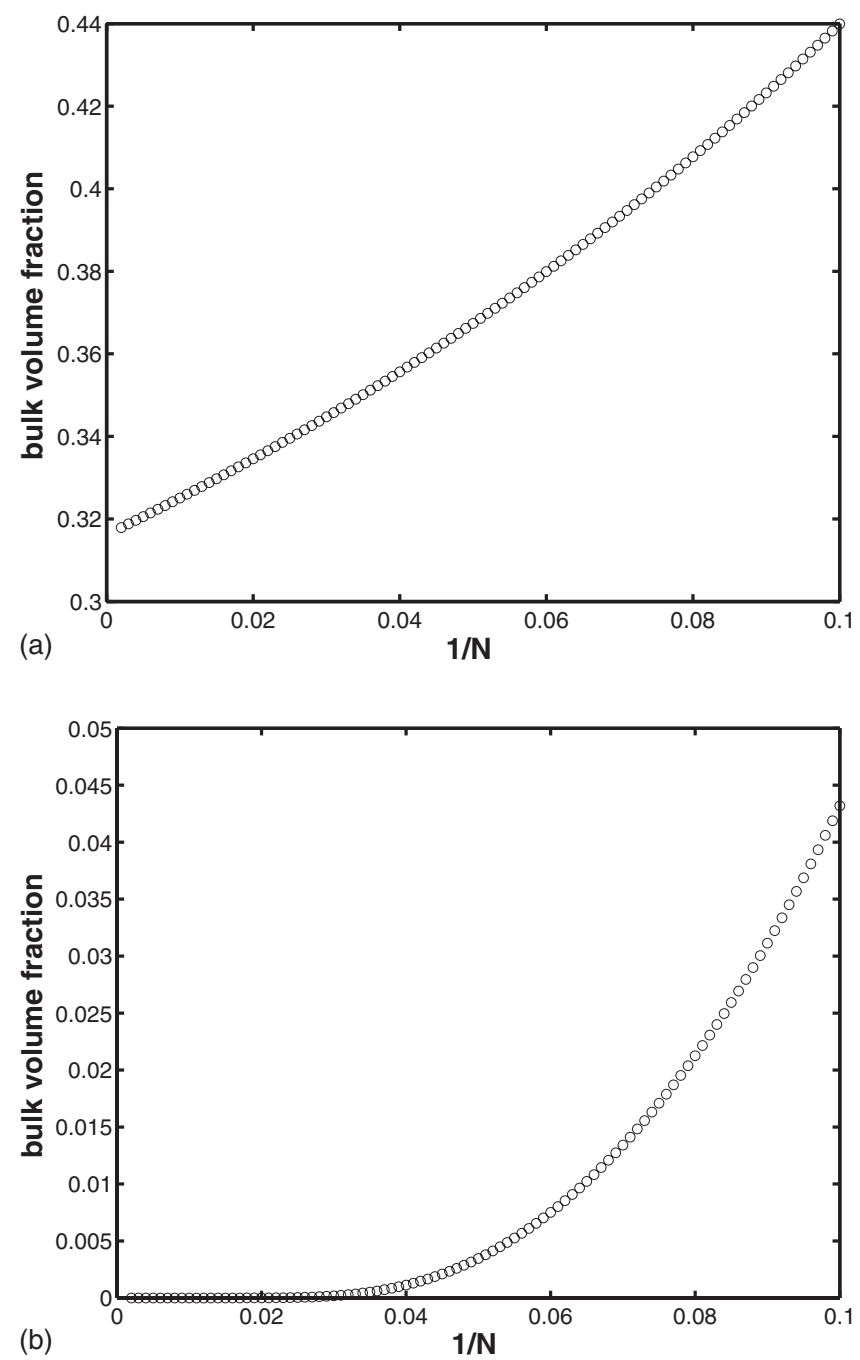

FIG. 3. Bulk volume fractions far from the interface of (a) solvent in polymer rich phase and (b) polymer in solvent rich phase as a function of inverse molecular-weight $1 / N$.

two-regime behavior of polymer surface tension with $\mathrm{MW}$ is indeed also predicted by SCFT for compressible systems. Figures 4 and 5 shows a number of different MW-dependent surface tensions for a range of compressibilities. In the regions of Fig. 5 where a linear dependence of inverse MW is found, there is consistently no discernible polymer in the solvent phase. Similarly, for regions where a $(1 / N)^{2 / 3}$ dependence is observed, there is always a non-negligible amount of polymer in the solvent phase.

It would be interesting to investigate further if systems with very large hole volume fractions (very high compressibilities) continue along the same trends as observed here. Quantitative predictions should also be possible if some optional simplifications of SCFT are removed, in particular the assumption of a common volume $\rho_{0}^{-1}$ for polymer segments, solvent volumes, and hole sizes. At higher molecularweights, the ground-state approximation for polymer configurational entropy is valid, and so the formalism should be reducible to something similar to density gradient theory, but with a more rigorous derivation. Given the quantitative success of phenomenological density gradient theory, a similar 


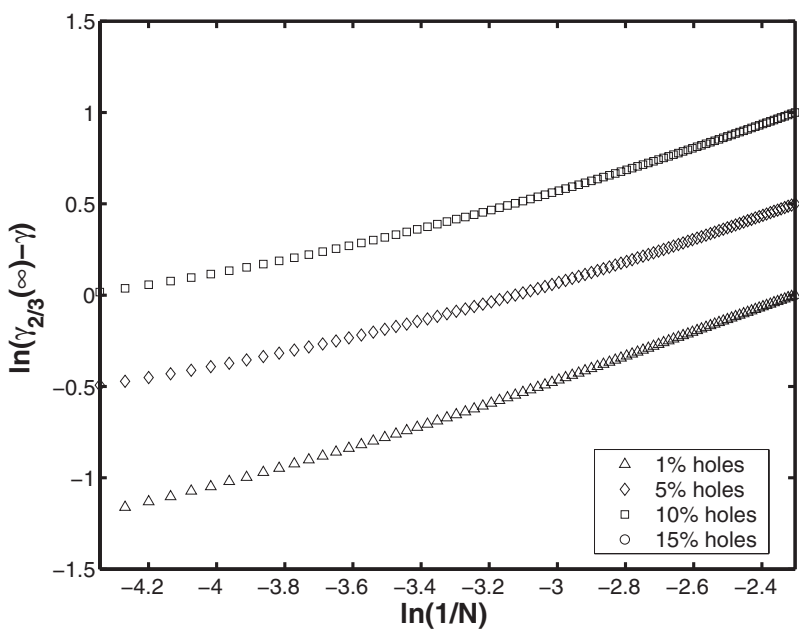

FIG. 4. Logarithmic plot of the dimensionless surface tension as a function of inverse molecular-weight for polymer plus solvent total global volume fractions of 0.99 (triangles), 0.95 (diamonds), 0.90 (squares), and 0.85 (circles). These correspond to hole volume fractions of $1 \%, 5 \%, 10 \%$, and $15 \%$, respectively. The surface tension is plotted as the logarithm of the difference between the dimensionless surface tension and an extrapolated infinite MW surface tension for a $2 / 3$ power relationship as discussed in the text. The results have also been shifted along the $y$ axis so that the smallest MW of each set falls at 0, 0.5, 1.0, and 1.5, respectively, to clearly show the results beside each other. The solid lines show a 2/3 slope.

quantitative success of the more elaborate microscopic, multicomponent SCFT approach is to be expected, and comparisons could be made between the two approaches. This can be studied in future work. It would also be interesting to see if the functional forms of low and high molecular-weight polymer surface tension can be derived using the differences between the thermodynamic components of the surface tensions and bulk surface tensions.

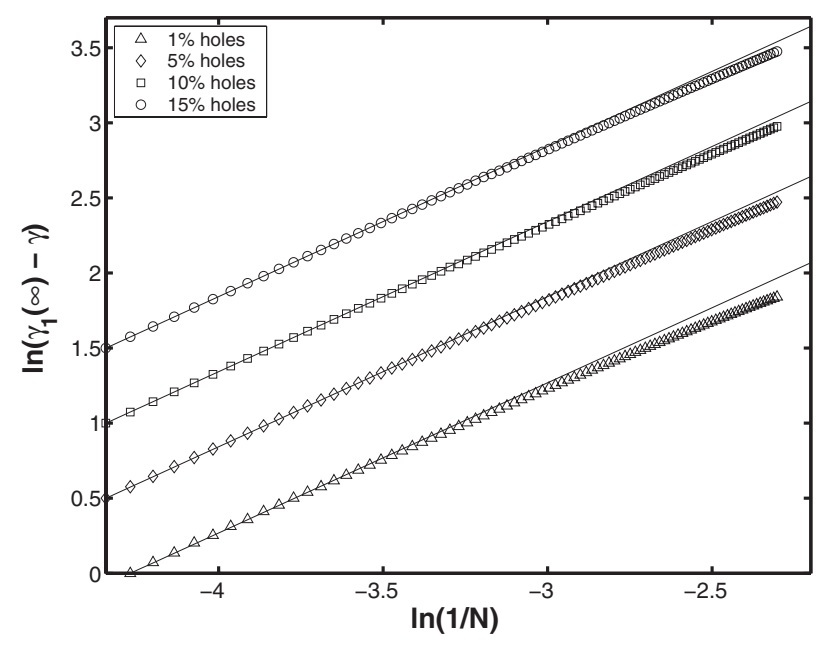

FIG. 5. Same data as Fig. 4 but plotted with an infinite molecular-weight surface tension based on an extrapolation for a linear power relationship. The results have been shifted along the $y$ axis so that the largest MW of each set falls at $0,0.5,1.0$, and 1.5 for $1 \%, 5 \%, 10 \%$, and $15 \%$ hole volume fractions, respectively, in order to clearly show the results beside each other.

In summary, we have demonstrated that mean-field exact results for polymer surface tension as a function of polymer molecular-weight can reproduce higher and lower MW behavior in agreement with experiments. We can explain the change in behavior between higher and lower MW in terms of the almost complete exclusion of polymer from the bulk solvent region. It is found that this explanation and prediction are also valid for compressible systems. These two experimentally observed domains of polymer surface tension with molecular-weight can be spontaneously reproduced with a single theory.

This work was supported by the Natural Sciences and Engineering Research Council of Canada (NSERC).
[1] D. Myers, Surfaces, Interfaces and Colloids: Principles and Applications (VCH, New York, 1991).

[2] H. Park, C. B. Park, C. Tzoganakis, and P. Chen, Ind. Eng. Chem. Res. 46, 3849 (2007).

[3] C. I. Poser and I. C. Sanchez, J. Colloid Interface Sci. 69, 539 (1979).

[4] E. Helfand, S. M. Bhattacharjee, and G. H. Fredrickson, J. Chem. Phys. 91, 7200 (1989).

[5] A. V. Ermoshkin and A. N. Semenov, Macromolecules 29, 6294 (1996).

[6] S. K. Kumar and R. L. Jones, Adv. Colloid Interface Sci. 94, 33 (2001).

[7] G. T. Dee and B. B. Sauer, Adv. Phys. 47, 161 (1998).

[8] G. T. Dee and B. B. Sauer, J. Colloid Interface Sci. 152, 85 (1992).

[9] G. T. Dee and B. B. Sauer, J. Colloid Interface Sci. 162, 25 (1994).

[10] R. A. L. Jones and R. W. Richards, Polymers at Surfaces and Interfaces (Cambridge University Press, New York, 1999).
[11] H. Park, R. B. Thompson, N. Lanson, C. Tzoganakis, C. B. Park, and P. Chen, J. Phys. Chem. B 111, 3859 (2007).

[12] K. M. Hong and J. Noolandi, Macromolecules 14, 1229 (1981).

[13] K. Binder, M. Müller, P. Virnau, and L. G. MacDowell, Adv. Polym. Sci. 173, 1 (2005).

[14] The subtracted bulk free energies are modified by volume proportionality factors in order to preserve conservation rules. See Eq. (15) of Ref. [11] and see Ref. [15].

[15] M. W. Matsen, in Soft Matter, edited by G. Gompper and M. Schick (Wiley-VCH, Weinheim, 2005), Vol. 1.

[16] D. G. LeGrand and G. L. Gaines, J. Colloid Interface Sci. 31, 162 (1969).

[17] In this work, qualitative trends are being examined, and so no direct numerical comparison with experiment is provided. Quantitative comparisons should be possible if some optional simplifications of SCFT are removed.

[18] M. W. Matsen and F. S. Bates, J. Chem. Phys. 106, 2436 (1997). 